\title{
Spatially Resolved Frequency Domain Phosphorescence Lifetime-Based Oxygen Sensing for Photodynamic Therapy
}

ARTICLE in PROCEEDINGS OF SPIE - THE INTERNATIONAL SOCIETY FOR OPTICAL ENGINEERING · JUNE 2008

DOWNLOADS

10

4 AUTHORS, INCLUDING:

Lothar Lilge

Princess Margaret Cancer Centre AND Univer..

220 PUBLICATIONS 2,607 CITATIONS

SEE PROFILE
VIEWS

17 


\title{
Spatially Resolved Frequency Domain Phosphorescence Lifetime-Based Oxygen Sensing for Photodynamic Therapy
}

\author{
Benjamin Lai ${ }^{\mathrm{a}}$, Mark Gurari ${ }^{\mathrm{b}}$, Wallace Wee ${ }^{\mathrm{c}}$, Lothar Lilge*a, \\ ${ }^{a}$ Department of Medical Biophysics, University of Toronto, Toronto, Ontario, Canada; \\ ${ }^{b}$ Division of Biophysics and Bioimaging, Ontario Cancer Institute, Toronto, Ontario, Canada; \\ ${ }^{\mathrm{c}}$ Department of Electrical and Computer Engineering, University of Toronto, Toronto, Ontario \\ Canada
}

\begin{abstract}
Photodynamic Therapy (PDT) is a minimally invasive treatment that uses a photosensitive drug into convert triplet state oxygen $\left({ }^{3} \mathrm{O}_{2}\right)$ to singlet oxygen $\left({ }^{1} \mathrm{O}_{2}\right)$ to destroy malignant tissue. A fiber-optic system based on frequency domain detection of phosphorescence quenching by ${ }^{3} \mathrm{O}_{2}$ is described which optically measures the distribution of ${ }^{3} \mathrm{O}_{2}$ in the treatment volume during PDT to permit adjustments of treatment parameters to improve outcome. A specially designed fiber optic probe containing phosphorescent sensors embedded along its length permit spatially resolved measurements. Each sensor is composed of a phosphorescent metalloporphyrin compound that emits a characteristic spectrum. Four candidate sensors with high absorption at the excitation wavelength of $405 \mathrm{~nm}$ and emission in the $650 \mathrm{~nm}$ to $700 \mathrm{~nm}$ region are considered. The dependence of phosphorescence lifetime $(\tau)$ on ${ }^{3} \mathrm{O}_{2}$ concentration is described by the linearized Stern-Volmer relationship as being inversely proportional. Determination of $\tau$, and hence ${ }^{3} \mathrm{O}_{2}$ concentration, is accomplished in the frequency domain by means of phase-modulation detection of the phosphorescence signal due to an amplitude modulated excitation. The $\tau$ 's of each sensor are recovered by performing global non-linear least squares fit on the measured phase and modulation index over a range of frequencies and wavelengths. With the $\tau$ of each sensor known, the oxygen concentration at each sensor's location can be determined with the Stern-Volmer relationship.
\end{abstract}

Keywords: Photodynamic Therapy, frequency domain lifetime measurement, metalloporphyrin, oxygen detection

\section{INTRODUCTION}

Photodynamic therapy is a promising treatment modality for treating both malignant conditions such as tumors [1] and non cancerous conditions like macular degeneration [2]. The treatment is based on activating photosensitive drugs that undergo type-II photochemical reactions with molecular oxygen in its triplet state $\left({ }^{3} \mathrm{O}_{2}\right)$, which results in the generation of highly reactive but short-lived singlet oxygen molecules $\left({ }^{1} \mathrm{O}_{2}\right)$ as shown in fig.1. Singlet oxygen can induce a biological response by directly killing off target cells via inducing apoptosis or necrosis, shut down tumor vasculature or increase inflammatory responses [3].

There are three interrelated components involved in PDT: treatment light dose, photosensitizer dose and molecular oxygen. As a result, determination of an actual PDT "dose" to be used for determination of the treatment's effectiveness is difficult. Various techniques of PDT dosimetry have been proposed including "explicit dosimetry", where light, photosensitizer and oxygen quantities are measured directly at selected points in the target volume [4].

The work presented here describes a direct optical measurement system for explicit dosimetry of ${ }^{3} \mathrm{O}_{2}$ based on phosphorescence lifetime $(\tau)$ quenching of metalloporphyrin molecules by ${ }^{3} \mathrm{O}_{2}$. A selection of candidate phosphor molecules are considered and placed on a multi-sensor fiber optic probe to enable spatially resolved ${ }^{3} \mathrm{O}_{2}$ detection. The emission signal is spectrally resolved and frequency domain phase-modulation detection at chosen wavelengths is performed. A non-linear least squares fit based on the multi-exponential decay model is used to recover $\tau$ which is used to calculate ${ }^{3} \mathrm{O}_{2}$ concentration via the Stern-Volmer relationship.

*llilge@uhnresearch.ca; phone +1 416 946-4501; fax +1 416 946-6529

Photonics North 2008, Réal Vallée, Michel Piché, Peter Mascher, Pavel Cheben, Daniel Côté, Sophie LaRochelle, Henry P. Schriemer, Jacques Albert, Tsuneyuki Ozaki, Eds., Proc. of SPIE Vol. 7099, 709928, (2008) - 0277-786X/08/\$18 · doi: 10.1117/12.806913 

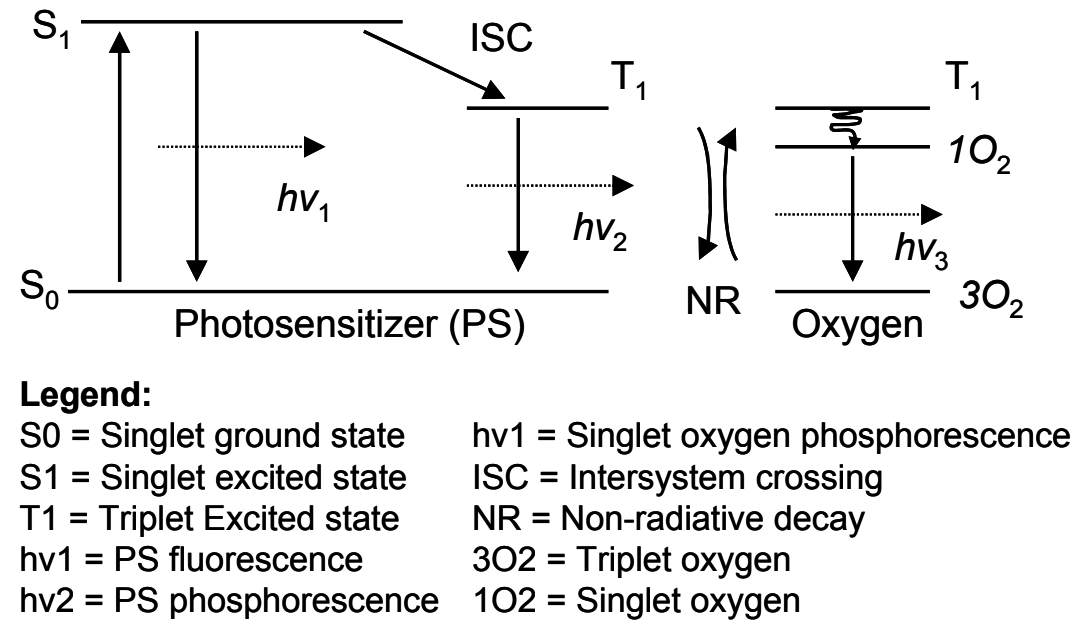

Fig. 1. Jablonski diagram of a type II photochemical reaction used in photodynamic therapy to generate singlet oxygen molecules.

\section{MATERIALS AND METHODS}

\subsection{Phosphor Selection and Preparation}

Phosphorescent metalloporphyrin compounds are selected for their emission sensitivity in the presence of oxygen and producing measureable $\tau$ 's as a function of ${ }^{3} \mathrm{O}_{2}$ concentration $\left(\left[{ }^{3} \mathrm{O}_{2}\right]\right)$. These substances belong to a large family of organic compounds called tetrapyrrols which include porphyrins that are widely used as photosensitizers in PDT [5]. As they are promoted to higher energy states by an excitation source, some molecules undergo a change in their electron configurations (spin flip) and cross over to the excited triplet state. Relaxation back to their grounded singlet state results in phosphorescence emission. The dipole transition, however, is quantum mechanically prohibited, and therefore requires significantly more time than singlet-singlet transitions for example and thus emissions can be readily detected in the time or frequency domains.

Four candidate phosphors (Frontier Scientific, Utah USA) have been chosen for their strong absorption in the dark blue range and large $\tau$ 's at zero ${ }^{3} \mathrm{O}_{2}$ in the microsecond ranges. Their measured optical characteristics and published known lifetimes in the absence of oxygen are listed in table 1.

Table 1. Absorption and emission maxima of candidate phosphors.

\begin{tabular}{|l|c|c|c|}
\hline \multicolumn{1}{|c|}{ Compound Name } & $\begin{array}{c}\text { Measured } \\
\text { absorption } \\
\text { maximum in } \\
\text { DMSO (nm) }\end{array}$ & $\begin{array}{c}\text { Measured } \\
\text { emission } \\
\text { maximum in } \\
\text { DMSO with } \\
405 \mathrm{~nm} \\
\text { excitation }(\mathrm{nm})\end{array}$ & $\begin{array}{c}\text { Published lifetimes } \\
\left.\text { at } 0 \%{ }^{3} \mathrm{O}_{2}\right] \text { in } \\
\text { solution } \\
(\mu \mathrm{s})\end{array}$ \\
\hline Pd meso-Tetra(4 carboxyphenyl) porphine "carboxy" & 418 & 697 & $690[6]$ \\
\hline Pd coproporphyrin III "copro" & 393 & 666 & $1100[7]$ \\
\hline Pd meso-Tetra(N-Methyl-4-Pyridyl) Porphine "Pyridyl" & 421 & 676 & $790[7]$ \\
\hline Pd meso-Tetra(4 sulfonatophenyl) porphine "sulfona" & 412 & 700 & $1000[7]$ \\
\hline
\end{tabular}

Triplet ground state molecular oxygen $\left({ }^{3} \mathrm{O}_{2}\right)$ can quench phosphorescence effectively. In the presence of ${ }^{3} \mathrm{O}_{2}$ the transition time to the singlet state decreases as energy is exchanged non-radiatively between a triplet-state excited 
phosphor molecule and ${ }^{3} \mathrm{O}_{2}$ through collision. As a result the $\tau$ decreases with increasing amounts of ${ }^{3} \mathrm{O}_{2}$. This process is described by the linear Stern-Volmer relationship [6], showing that $\tau$ and the amount of ${ }^{3} \mathrm{O}_{2}$ present is inversely proportional:

$$
\tau_{0} / \tau=1+\tau_{0} k_{q}\left[{ }^{3} O_{2}\right]
$$

where

$$
\begin{aligned}
& \tau_{0}=\text { the phosphorescence lifetime in the absence of oxygen } \\
& \tau=\text { the lifetime at an oxygen concentration }\left[{ }^{3} \mathrm{O}_{2}\right] \\
& k_{q}=\text { the quenching constant. }
\end{aligned}
$$

The quenching constant is determined by measuring $\tau$ in the absence of ${ }^{3} \mathrm{O}_{2}$ and at a known $\left[{ }^{3} \mathrm{O}_{2}\right]$ level, during a calibration process. This relationship is then used later to estimate the amount of oxygen present surrounding the phosphor by measuring its $\tau$.

For sensor preparation, each of the phosphors is dissolved into dimethyl sulfoxide (DMSO) to yield $1 \mathrm{mM}$ concentrations before being mixed in equal parts with ultraviolet (UV) curing epoxy (Dymax 141-M, Connecticut USA) achieving a $0.5 \mathrm{mM}$ final concentration. This mixture is used for the validation measurements and also be applied to the fiber probe as one of the sensors.

\subsection{Oxygen sensing probe}

The probe design chosen for this system has been previously described by Pomerleau-Dalcort et al [7]. A schematic diagram of the probe design is shown in fig 2a. Excitations and emissions from the embedded phosphors are transferred via the optical fiber. Because each groove contains a particular phosphor with its own unique emission spectrum, the detected optical signal will be a superposition of all individual sensors. Therefore the spatial distribution of oxygen concentrations can be determined by the spectrally resolved $\tau$ 's of each known sensor.

Silica multimode fibers (Thorlabs FG200LCC, New Jersey USA) are selected for the construction of the probes. These fibers are chosen for their high transmission in the visible and near-infrared (NIR) range and their mechanical strength. The fibers contain an outer buffer layer, a silica cladding layer in the middle and a silica core. For the test fiber grooves $250 \mu \mathrm{m}$ long are cut through the buffer, $1 \mathrm{~cm}$ apart, and the fiber immersed in hydrofluoric acid (HF) for 24 minutes to completely remove the cladding. The phosphor/glue mixture is placed onto the exposed core and allowed to harden under a spot UV curing source for 5 seconds and followed by heat annealing at $60 \mathrm{C}$ for 24 hours. A photograph of the constructed probe with sensors applied to the grooves is shown in fig. $2 \mathrm{~b}$.
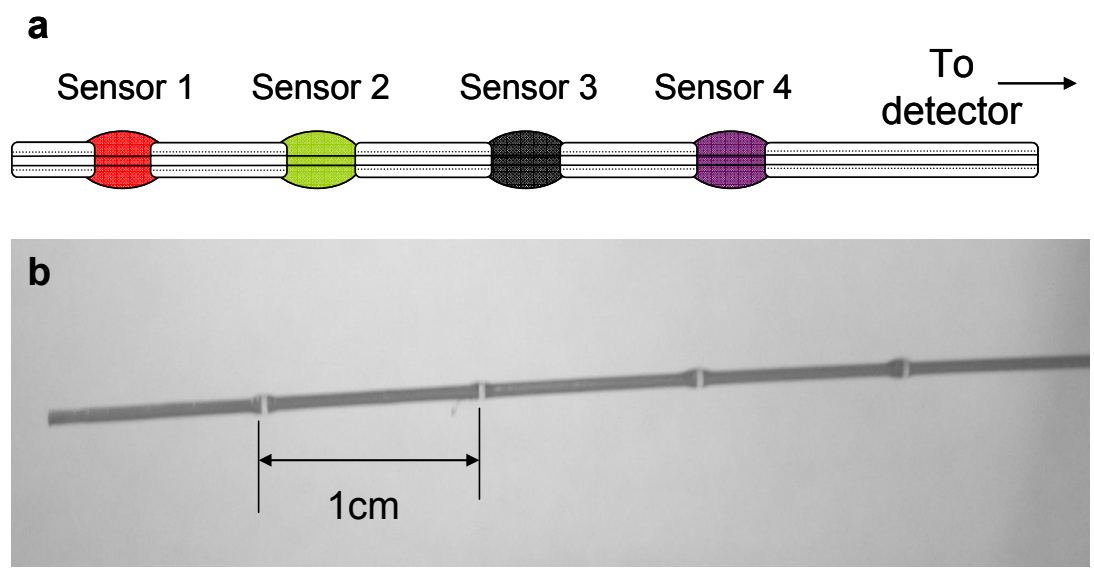
Fig. 2. (a) Schematic drawing of silica fiber probe with sensors placed over grooves. (b) Photograph showing a fabricated test probe with sensors placed over grooves

\subsection{Frequency domain method for determining $\tau$ of a single phosphor}

Phosphorescence lifetime can be determined in the frequency domain by means of phase-modulation detection. This technique has been well established in measuring fluorescence lifetimes of organic and inorganic compounds [8].

The excitation source is intensity modulated with a sinusoidal pattern at a desired frequency and modulation. It is described by $E(t)=a+b \sin (\omega t)$. Where $a$ is the DC intensity, $b$ is the AC amplitude, and $\omega$ is the angular modulation frequency in radians per second. The resultant phosphorescence emission is intensity modulated at the same frequency as the excitation source, but with a phase delay and a different modulation depth. The phosphorescence behaviour is described by $P(t)=A+B \sin (\omega t+\phi)$ where $A$ and $B$ are the DC and AC components respectively, and the extra term $\varphi$ is the phase delay compared to $E(t)$.

In phase-modulation detection, a unitless quantity called the modulation index $m$, is defined by comparing the DC and AC components of both signals, $m=\frac{B / A}{b / a}$, while the phase of the two signals is determined by examining the cross correlation between $E(t)$ and $P(t)$.

Phosphorescence intensity decays exponentially following a first order behaviour. Its response to an infinitely short pulse excitation is

$$
P(t, \lambda)=\alpha(\lambda) e^{-\frac{t}{\tau}}
$$

Where

$$
\begin{array}{ll}
\alpha(\lambda) & =\text { wavelength dependent pre-exponential coefficient } \\
\tau & =\text { decay lifetime, time to reach } 1 / e \text { of the initial intensity }
\end{array}
$$

Note that $\tau$ is independent of wavelength and therefore phosphorescence intensity decays at the same rate throughout the entire emission spectrum.

If only a single phosphorescence source is considered, the $\tau$ can be recovered by either detecting the phase or modulation index at a chosen frequency $\omega$ :

$$
\tan \left(\phi_{\omega}\right)=\omega \tau
$$

and

$$
m_{\omega}=\left(1+\omega^{2} \tau^{2}\right)^{-1 / 2}
$$

The $\tau$ of each candidate phosphor is measured individually by first placing the phosphor/glue mixture on a glass microscope slide, spin coated to approximately $100 \mu \mathrm{m}$ thickness and allowed to polymerize. Phase information is taken for each phosphor in environments containing $0 \%, 14.7 \%$ (from calibrated compressed gas canisters) and 21\% oxygen (air). Excitation frequencies from $100 \mathrm{~Hz}$ to $1000 \mathrm{~Hz}$ stepped through at $100 \mathrm{~Hz}$ intervals are used and a corresponding $\tau$ is calculated using equation 3 from the phase measurements at each frequency.

\subsection{Frequency domain method for determining $\tau$ for multiple phosphors}

When multiple phosphors are considered the detected phosphorescence signal is the sum of each emission source. A global non-linear least squares fit is required to estimate the $\tau$ 's of each phosphor [8]. A software package called CFS_LS written by the Center for Fluorescence Spectroscopy at the University of Maryland School of Medicine has 
been adapted to implement the least squares estimation. The software requires both the phase and modulation index of the detected signal over a range of chosen frequencies and wavelengths to compute the contributing $\tau$ and $\alpha$ of each sensor based on a multi-exponential decay model.

\subsection{Hardware Components}

The system block diagram of the frequency domain detection system is presented in fig. 3. A 50:50, 2x2 optical coupler is used to direct excitation light to the sample and emissions to the detectors.

The excitation source for the system is a $405 \mathrm{~nm}$ laser diode (LD). Modulation of the source is accomplished by connecting a function generator (National Instruments NI5401, Texas USA) to the LD driver (Wavelength Electronics WLD3393, Montana USA). The laser's output is then sent into an optical coupler on port 1. A reference photomultiplier tube (PMT) (Hamamatsu 7827, Hamamatsu Japan) at port 3 equipped with a bandpass filter centered at $405 \mathrm{~nm}$ monitors the modulated excitation. The excitation light is also delivered to port 4 to induce phosphorescence in the sample within the gas chamber. The phosphorescence emission enters port 4 and reaches port 2 for detection. If a single sensor is considered, a single PMT would suffice for detection. A $650 \mathrm{~nm}$ Long Pass filter is installed to the detection PMT (port 2) to eliminate shorter wavelengths from limiting the dynamic range of the PMT. Data collection is accomplished via a 16-channel acquisition card (National Instruments 6036E, Texas USA).

For multiple sensors, a spectrometer (ORIEL MS127i, California USA) equipped with a 32 channel PMT is used to spectrally resolve the emission signal before it is acquired for analysis.

A gassing chamber was built to house the samples in order to control the concentration of oxygen exposed to the sensors. Known $\left[{ }^{3} \mathrm{O}_{2}\right]$ concentrations $0 \%$ (purging with nitrogen), $14.7 \%$ (calibrated compressed gas), $21 \%$ (air) are used to fill the chamber. A small opening $5 \mathrm{~mm}$ diameter is present for excess gas to escape, and maintaining a partial pressure at 1 atmosphere.

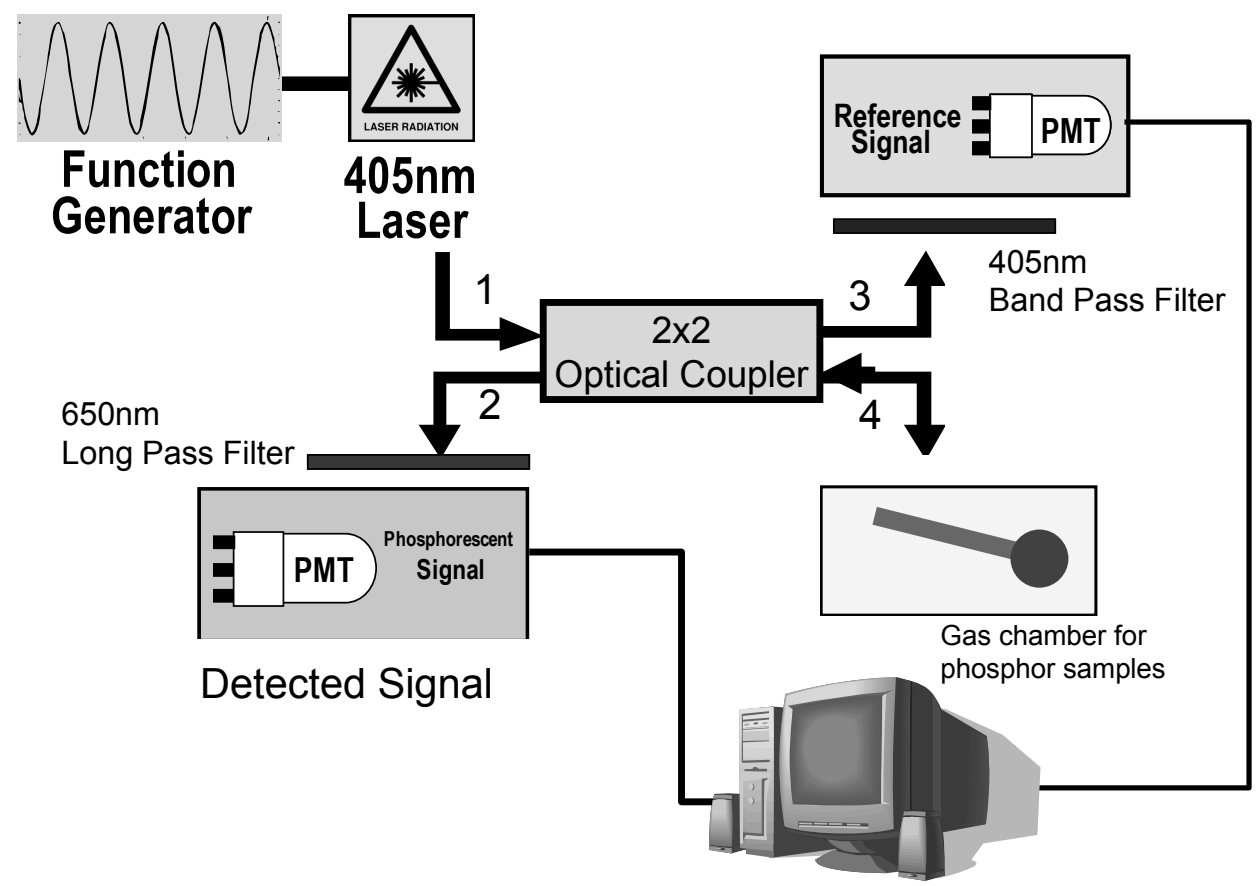

Fig. 3: System block diagram of the frequency domain measurement system. Excitation light enters the coupler via port 1, and exits port 3 where it is tracked. At port 4 the excitation light irradiates the sample to induce phosphorescence. Phosphorescence emissions enter port 4 which exits the coupler at port 1 and 2 . The phosphorescence is collected at port 2 with a PMT equipped with a $650 \mathrm{~nm}$ long pass filter. This PMT is replaced with a spectrometer when the $\tau$ 's of multiple phosphors are needed. 


\subsection{Software components}

LabView from National Instruments is used to control the data acquisition card, function generator and for calibration of the phosphors. Additional processing of acquired data is also performed in LabView including low-pass filtering (to remove high frequency electronic noise), phase-modulation detection and formatting the data for least squares analysis. Once the $\tau$ information is gathered, it will also generate the $\left[{ }^{3} \mathrm{O}_{2}\right]$ information using the Stern-Volmer relationship.

The LabView software calls upon the least squares estimation program, CFS_LS, to estimate $\tau$. This software is intended for estimating fluorescence lifetimes and the pre-exponential coefficients by means of non-linear least squares fit on measured phase-modulation fluorescence data. The software uses a multi-exponential decay model as the mathematical basis for the fit. It assumes that each phosphor decays exponentially, independent of wavelength, as described by equation 2. Typically modulation frequencies and lifetimes are in the RF (typically $100 \mathrm{MHz}-1 \mathrm{GHz}$ ) and nanosecond ranges, respectively for fluorescence lifetime measurements. However for phosphorescence lifetimes ranging in several hundred microsecond range, the modulation frequency in the $100-1000 \mathrm{~Hz}$ range is sufficient to observe any frequency dependant phase shifts.

Software validation is performed to assess the CFS_LS's abilities to work at lower frequencies, and its ability to reliably recover multiple $\tau$ 's. Simulated phase-modulation data for 1, 2 and 3 sensors cases are generated, according to [8]. The data set is made by selecting desired $\alpha$ and $\tau$ to generate a set of corresponding phase and modulation data at each modulation frequency of interest $(100 \mathrm{~Hz}$ to $1000 \mathrm{~Hz})$. For the 3 -sensor case, additional data sets representing phasemodulation data acquired at different wavelengths (representing changes in the pre-exponential coefficient) are created.

\section{RESULTS AND DISCUSSION}

\subsection{Validation of Non-linear Least Squares Fitting Software}

The CFS_LS output information includes the recovered $\tau$, and the phase and modulation index derived based on this recovered value. It is then plotted together with the input data shown in fig. 4. The software also calculates a quantity called the Square Root of Variance (SRV), a quick way to examine the goodness of fit. It is defined as the sum of the squared residuals (deviations between measured and calculated values) divided by the number of degrees of freedom (the number of data points minus the number of parameters being estimated).

The recovered $\tau$ 's for 1 and 2 sensor scenarios result with an SRV of less than 0.01 with one iteration of the software, with initial guesses that are $30 \%$ from the actual values.

For the 3-sensor case, initial $\tau$ guesses $30 \%$ from the actual $\tau$ 's are also used. Approximately 5 iterations are required to achieve an SRV of less than 0.05, which corresponds to an error of around $1 \%$, between the measured and calculated phase-modulation data when the chosen $\tau$ 's used are selected to be $200 \mu \mathrm{s}$ apart $(100 \mu \mathrm{s}, 300 \mu \mathrm{s}$ and $500 \mu \mathrm{s})$. Figure 4 shows the fit between calculated and measured phase-modulation data for the decomposition of 3 sensors and table 2 summarizes the results for all 3 scenarios.

Table 2: Comparison of predefined and recovered $\tau$ with simulated data to validate CFS_LS

\begin{tabular}{|l|c|c|c|c|c|c|c|}
\hline \multirow{2}{*}{ Scenario } & \multicolumn{3}{|c|}{ Predefined $\tau$ (in $\mu \mathrm{s})$} & \multicolumn{2}{c|}{ Recovered $\tau$ (in $\mu \mathrm{s})$} & \multicolumn{2}{c|}{$\begin{array}{c}\text { \% Avg. } \\
\text { Error }\end{array}$} \\
\cline { 2 - 7 } & $\tau 1$ & $\tau 2$ & $\tau 3$ & $\tau 1$ & $\tau 2$ & $\tau 3$ & \\
\hline 1 Sensor & 500 & $\mathrm{X}$ & $\mathrm{X}$ & 500 & $\mathrm{X}$ & $\mathrm{X}$ & 0 \\
\hline 2 Sensors & 500 & 100 & $\mathrm{X}$ & 499.8 & 99.7 & $\mathrm{X}$ & 0.4 \\
\hline 3 Sensors (5 iterations) & 500 & 100 & 300 & 499.1 & 98.9 & 295.3 & 0.9 \\
\hline
\end{tabular}




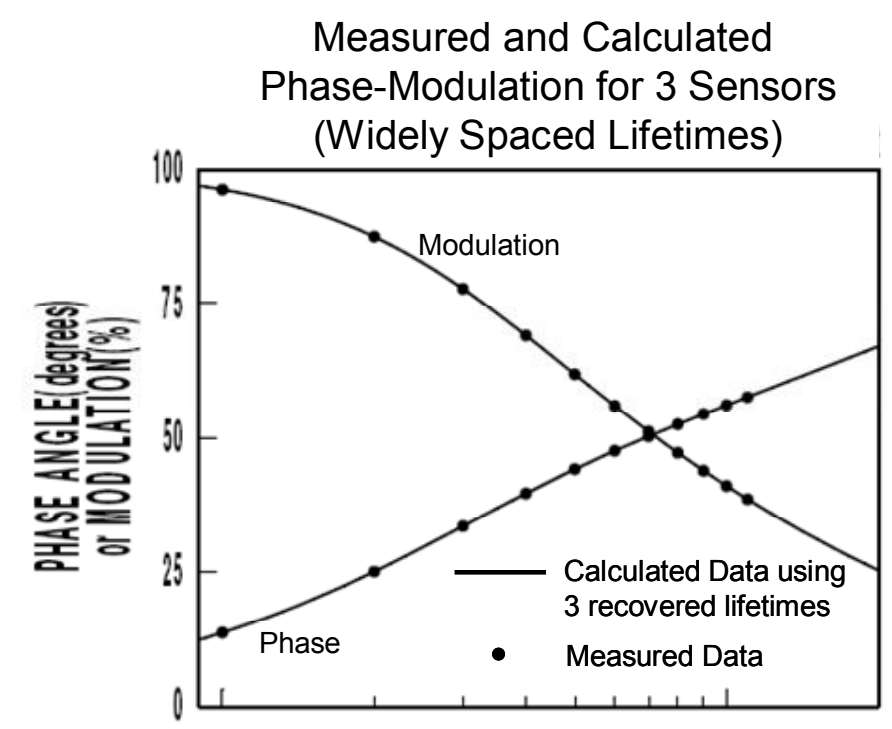

Fig. 4: CFS_LS plot of the measured phase-modulation data (points) and calculated phase-modulation based on the estimated $\tau$ (solid line), showing a fit of an SRV $=0.05$ with simulation data.

\subsection{Validation of hardware and software setup with single phosphor measurements}

Phase and modulation measurements using the LabView program with the samples inside the gas chamber are performed as described previously. Ideally the recovered $\tau$ based on the measured phase and modulation index at all frequencies using equations 3 and 4 respectively should be identical. However it is never the case in a real system. For example column 3 of table 3 shows the range of $\tau$ 's recovered using equation 3 based on the measured phase. The corresponding ranges of $\left[{ }^{3} \mathrm{O}_{2}\right]$ calculated using equation 1 for each phosphor at $21 \%\left[{ }^{3} \mathrm{O}_{2}\right]$, based on $k_{q}$ derived using the average $\tau$ at $21 \%$ and $0 \%\left[{ }^{3} \mathrm{O}_{2}\right.$, are also shown.

Table 3: $\tau$ measured derived on phase measurements at $21 \%{ }^{3} \mathrm{O}_{2}$. Good matching is seen at the chosen frequencies for Pyridyl, though uncertainties in measured $\left[{ }^{3} \mathrm{O}_{2}\right]$ is much large for the remaining samples

\begin{tabular}{|c|c|c|c|c|}
\hline Sample & $\begin{array}{c}\text { Quenching } \\
\text { constant } \\
k_{q}\left(\left[\mathrm{O}_{2}\right]^{-1} \mathrm{~s}^{-1}\right)\end{array}$ & $\begin{array}{c}\text { Average } \tau \\
(\mu \mathrm{s})\end{array}$ & $\begin{array}{c}\text { Standard } \\
\text { deviation } \\
\text { from average } \\
(\mu \mathrm{s})\end{array}$ & $\begin{array}{c}\text { Corresponding } \\
{\left[{ }^{3} \mathrm{O}_{2}\right] \text { range }} \\
(\%)\end{array}$ \\
\hline Pyridyl & 9.7 & 784 & 60 & $17.5-24.5$ \\
\hline Carboxy & 115.5 & 269 & 68 & $14.6-31.1$ \\
\hline Copro & 78.9 & 393 & 134 & $12.6-36.8$ \\
\hline
\end{tabular}

Good matching for Pyridyl is achieved because it has the strongest emission intensity of the four phosphorescent compounds. At $21 \%\left[{ }^{3} \mathrm{O}_{2}\right]$ the average measured $\tau$ is $784 \mu$ s with a standard deviation of $60 \mu$ s for the range of frequencies in use. This measurement step validates the capability of the current setup and detection method for $\left[^{3} \mathrm{O}_{2}\right]$ detection.

However, less satisfactory outcomes arise with the remaining phosphor samples. An extremely weak emission signal was detected for Sulfona and was not sufficient to properly measure its lifetime. For the remaining two samples the 
expected behaviour of the phase relation between the emission and reference signal should increases along with the modulation frequency. The observed measured phases for these phosphors fall as the modulation frequency increases. As the modulation index goes down with increasing frequency, this implies a drop in the phosphorescence intensity and thus SNR. It becomes more difficult to reliably determine the phase shift as a result of a diminished emission signal. A large variation of calculated $\tau$ values result, leading to the uncertainties in the corresponding $\left[{ }^{3} \mathrm{O}_{2}\right]$ levels being large as well. Therefore further investigation is required to improve emission signal quality of these phosphors and their detection without negatively affecting the responsivity of the sensor.

\section{ACKNOWLEDGEMENTS}

The authors would like to thank Kevin Perry and Walsh Medical for precision machining of the silica fibers used for the probes, Bastian Bräuer, Johannes Schleusener, Yi Yao and Wayne Chau for the hardware and software support. The authors would also like to acknowledge NIH contract POIC1743892 and CIHR contract 67895 for funding this project.

\section{REFERENCES}

[1] Dolmans, D.E.J.G.J., Fukumura, D., Jain, R.K., "Photodynamic therapy for cancer", Nature Reviews Cancer 3, 380387 (2003)

[2] Khurana, M., Karotki, A., Moriyama, E.H., Akens, M.K., Wilson, B.C., "Development and Application of Biological Techniques to Two-Photon Photodynamic Therapy", Proc. SPIE 6796, 679600 (2007)

[3] Castano, A.P., Mroz, P., Hamblin, M.R., "Photodynamic therapy and anti-tumour immunity" Nature Reviews Cancer 6, 535-545 (2006)

[4] Wilson, B.C., Patterson, M.S., Lilge, L., "Implicit and explicit dosimetry in photodynamic therapy: a new paradigm", Lasers Med Sci 12, 182-199 (1997)

[5] Papkovsky, D.B., O’Riordan, T.C., "Emerging Applications of Phosphorescent Metalloporphyrins", J Fluoresc. 215 (4), 569-84 (2005)

[6] Lo, L. W., Koch, C. J., and Wilson, D. F., "Calibration of oxygen-dependent quenching of the phosphorescence of Pd-mesotetra (4-carboxyphenyl) porphine: A phosphor with general application for measuring oxygen concentration in biological systems", Anal. Biochem. 236(1), 153-160 (1996)

[7] Vanderkooi, J. M., Maniara, G., Green, T. J., Wilson, D. F., "Anoptical method formeasurement of dioxygen concentration based upon quenching of phosphorescence", J. Biol. Chem. 262(12), 5476-5482 (1987)

[8] Stern, O., Volmer M., "Uber die ablingungszeit der fluoreszenz", Physik. Zeitschr 20, 183-188 (1919)

[9] Pomerleau-Dalcourt, N., Lilge, L., "Development and characterization of multi-sensory fluence rate probes", Phys Med Biol. 51(7), 1929-40 (2006)

[10] Lakowicz, JR, Principles of Fluorescence Spectroscopy, 2nd Ed., (Plenum, New York, 1999) 